\title{
Predicting Early Failure of Quantum Cascade Lasers During Accelerated Burn-in Testing Using Machine Learning
}

\section{A. Cagri Aydinkarahaliloglu ( $\square$ aaydinka@nd.edu )}

University of Notre Dame

Shashank Jatar

AdTech Optics (United States)

\section{Xiaojun Wang}

AdTech Optics (United States)

Mary Fong

AdTech Optics (United States)

Vijay Gupta

University of Notre Dame

Mariano Troccoli

Evolution Photonics Inc

Anthony J. Hoffman

University of Notre Dame

\section{Research Article}

Keywords: Support vector machines, quantum cascade lasers, Catastrophic failure

Posted Date: March 2nd, 2022

DOI: https://doi.org/10.21203/rs.3.rs-1393768/v1

License: (9) This work is licensed under a Creative Commons Attribution 4.0 International License.

Read Full License 


\section{Abstract}

Device life time is a significant consideration in the cost of ownership of quantum cascade lasers (QCLs). The life time of QCLs beyond an initial burn-in period has been studied previously; however, little attention has been given to predicting premature device failure where the device fails within several hundred hours of operation. Here, we demonstrate how standard electrical and optical device measurements obtained during an accelerated burn-in process can be used in a simple support vector machine to predict premature failure with high confidence. For every QCL that fails, at least one of the measurements is classified as belonging to a device that will fail prematurely-as much as 205 hours before the actual failure of the device. Furthermore, for devices that are operational at the end of the burn-in process, the algorithm correctly classifies all the measurements. This work will influence future device analysis and could lead to insights on the physical mechanisms of premature failure in QCLs.

\section{Introduction}

Quantum cascade lasers (QCLs) are electrically-injected semiconductor lasers that produce photons in the mid-infrared $(\lambda \sim 3-30 \mu \mathrm{m})$ and terahertz $(\lambda \sim 30 \mu \mathrm{m}-3 \mathrm{~mm})$ portions of the electromagnetic spectrum. In contrast to diode lasers, which produce photons via electron-hole recombination across a semiconductor bandgap, QCLs generate photons via intersubband transitions-electronic transitions between the quantized energy levels of quantum well heterostructures ${ }^{1}$. Many of the characteristics of QCLs can be tailored via the quantum design of the heterostructure, and recent progress has resulted in expanded spectral coverage and significant improvements in their overall performance ${ }^{2-9}$. These improvements have enabled or improved the use of QCLs in a growing number of applications in trace gas sensing, imaging, and defense ${ }^{10-12}$. However, their broad use in other applications, particularly those that involve integration into complex systems, can be limited due to concerns over premature device failure. Such premature device failure has been observed in practice as discussed in more detail below, is ill-understood from a mechanistic point of view, and drastically increases the cost of ownership of QCLs.

There have been several studies on the life time of QCLs under various testing conditions ${ }^{13-16}$. The average life time of QCLs has been estimated as 809 thousand hours when operated at a heat sink temperature of $25^{\circ} \mathrm{C}^{15}$. A major challenge in estimating the life time of QCLs is the low failure rate of the device past an initial operating period. Indeed, in much of the previous life time studies, the tested QCLs exhibit two timescales for failure: within several hundred hours of operation or after thousands of hours of operation; those studies focus on lasers that survive the initial operating period ${ }^{16-18}$. Premature failure refers to failures within the shorter timescale. Identifying QCLs that fail prematurely is typically accomplished using laser burn-in processes where the performance of the QCLs under test are monitored over time. This requires establishing a burn-in process that is sufficiently long in duration to observe the premature failure of devices. While longer burn-in times can increase the likelihood of observing premature failure, doing so can be costly and time-consuming. Additionally, it is not always possible to identify lasers that will fail prematurely after the conclusion of the initial burn-in period as the 
mechanisms for premature failure and its manifestation on the operational characteristics of the QCLs are not known.

An alternative approach to existing methods is to develop decision algorithms that use measured optical and electrical characteristics of QCLs to predict which lasers are likely to fail. Since the physical mechanisms leading to device failure are yet unknown, we do not know precisely which characteristics are predictive of device failure and in what regimes of values. However, as we show in this paper, a machine learning approach can identify relevant signatures of failures in these characteristics and reliably predict imminent device failure up to 205 hours in advance. This is an interesting and a novel finding because it enables fast and reliable identification of QCLs that are likely to fail, reducing the resources expended on the entire burn-in process.

Machine learning $(M L)$ is the study of improving systems automatically through experience ${ }^{19}$. ML has been used to determine or predict a particular outcome given specified input data in various applications from computer vision to speech recognition to natural language processing ${ }^{20-22}$. There are a variety of approaches to ML. In supervised ML, the objective is to develop a mapping function between the input and output data based on example input-output pairs. Support vector machines (SVMs) are a powerful means to implement this supervised ML paradigm. The objective of a SVM is to build and train a model with two classes using a set of training data consisting of a set of input data labeled with the correct class to which the data belong. The trained model is used to assign input data with an unknown class label to the correct class. During training, the SVM maps the training data to points in an N-dimensional space, where the dimensions are used to represent features of the dataset. Since the correct class for each training data point is known, the SVM can then calculate a hyperplane in the $\mathrm{N}$-dimensional space that maximizes the distance between the set of data points belonging to one class from the set belonging to the other class. Once training is complete, new data is mapped onto the same $\mathrm{N}$-dimensional space and classified using the hyperplane that has been learned ${ }^{23,24}$. Successful use of SVMs has been demonstrated in various applications, including milling chatter detection ${ }^{25}$, recognizing complex machining conditions ${ }^{26}$, gene selection for cancer classification ${ }^{27}$, and fault detection ${ }^{28,29}$.

In this work, we use a SVM to identify devices that will fail prematurely using conventional measurements of the electrical and optical characteristics of QCLs obtained during accelerated burn-in testing ${ }^{30}$. We develop an approach for parametrizing the electrical and optical measurements and use those results to train and test a SVM model. The trained model predicts failure as much as 205 hours before the observed premature failure of a QCL. Importantly, the SVM never identifies a device that does not fail as one that will. Such an SVM can enable a significant reduction in QCL manufacturing costs by identifying devices that are likely to experience premature failure early in the burn-in process, reducing the time spent on burn-in testing for those devices. Additionally, the fact that failure can be predicted using observations up to 200 hours in advance will enable future research into the mechanisms of premature failure in QCLs.

\section{Methods}




\section{Experimental Setup}

We test nine high-performance, Fabry-Perot QCLs using an accelerated burn-in process where the lasers are operated in continuous wave (CW) mode at an elevated heat sink temperature. The QCLs are from different portions of a single epitaxial wafer grown by metal organic chemical vapor deposition and therefore share the same multiple quantum well heterostructure design. The lasers are processed as buried heterostructures using $\mathrm{InP}$ regrowth and mounted epitaxial-side down on CuW C-mounts for efficient heat extraction. The QCLs are all $5 \mathrm{~mm}$ long, but their ridge width varies. Two identical testing stages are used for the accelerated burn-in testing. Each stage uses a temperature-controlled thermoelectric cooler mounted on a water-cooled base. The stages are both equipped with two thermopiles; thus, four QCLs (two per stage) can undergo the accelerated burn-in process simultaneously as depicted in Fig. 1.

During the accelerated burn-in process, the QCLs are operated in CW mode at $80 \%$ of their peak CW optical power at a heat sink temperature of $313 \mathrm{~K}$. Measurements of the $\mathrm{CW}$ current, voltage, and optical power are recorded every minute. Here, these frequent measurements are only used to determine when a device fails, which is indicated by measuring no output optical power from the biased device. Continuous wave light-current-voltage (LIV) measurements are performed approximately every 2.5 hours where the current, voltage, and output optical power are measured as the CW current is swept in $10 \mathrm{~mA}$ steps from zero to the operating point ( $80 \%$ of the peak output power). The planned burn-in time for seven of the QCLs is 150 hours, but there are differences in the overall test length due to starting and stopping the burn-in process manually. Two of the devices fail before the end of the planned burn-in procedure. Two additional QCLs undergo longer accelerated burn-in testing that is planned for 250 hours. During the burnin process for these devices, the interval between CW LIV measurements is increased and ranges from 10 to 48 hours between measurements.

Figure 2 shows every measured LIV for all nine QCLs obtained during the accelerated burn-in processes. The devices labeled $G_{N}(N=1 \ldots 5)$ indicate QCLs that are operational at the end of the burn-in testing. The devices labeled $\mathrm{B}_{\mathrm{N}}(\mathrm{N}=1 \ldots 4)$ indicate QCLs that failed during the burn-in testing. In Fig. 2, we omit measurements after device failure for devices B1 to B4. This data is also excluded when training and testing the SVM. Importantly, for the measurements shown in Fig. 2, there is no easily apparent distinction between the LIVs of devices that remain operational and devices that fail. However, we will show that incorporating the LIV measurements into SVMs can predict premature failure of the QCLs with very high accuracy.

We incorporate the LIV measurements into a SVM by extracting features from the measurements that express the significant characteristics of the data. The parameters are extracted automatically using custom software that generates a feature matrix for each LIV. Examples of extracted features include the wall-plug efficiency (WPE) at peak optical power; laser threshold current density; applied voltage at lasing threshold; maximum output optical power; slope of optical power versus current density at several operating points; and differential resistance (slope of voltage versus current density) at several operating 
points. In total, we extract 28 features describing each LIV measurement. All of the features are listed in Table 1. Here, $V$ is the voltage; $P$ is the measured optical power, $P_{\max }$ is the peak optical power measured from one facet, $J$ is the current density, and $R$ is the differential resistance.

Table 1: Features used in the SVM model.

\begin{tabular}{|c|c|c|c|c|c|c|}
\hline At $\mathbf{P}_{\text {th }}$ & At 1.1. $_{\text {th }}$ & ${\text { At } \mathbf{P}_{\max }}$ & Minimum & Maximum & Mean & Standard Deviation \\
\hline $\mathrm{V}, \mathrm{J}$ & $\mathrm{V}$ & $\mathrm{V}$ & & & & \\
\hline$d P / d t$ & $d P / d t$ & $d P / d t$ & $d P / d t$ & $d P / d t$ & $d P / d t$ & $d P / d t$ \\
\hline$d V / d t$ & $d V / d t$ & $d V / d t$ & $d V / d t$ & $d V / d t$ & $d V / d t$ & $d V / d t$ \\
\hline$d R / d t$ & $d R / d t$ & $d R / d t$ & $d R / d t$ & $d R / d t$ & $d R / d t$ & $d R / d t$ \\
\hline $\mathrm{R}$ & & & $\mathrm{R}$ & $\mathrm{R}$ & & \\
\hline
\end{tabular}

\section{Training and Testing}

To predict premature device failure, we use the 28 features in a SVM with a radial basis function (RBFSVM) kernel as the overall accuracy and number of false negatives was superior compared to the linear SVM. The training and testing of the model consist of two stages. In the initial stage, the model is trained and tested with data from the first set of devices with planned burn-in times of 150 hours $\left(G_{i} ; i=1 \ldots 5, B_{j} ;\right.$ $=1,2$ ). During training and testing, the LIVs of a single device are categorized according to the operational status of the device at the end of the burn-in period. For a device that fails during testing, all the LIVs are categorized as belonging to a failed device. Likewise, for a device that is operational at the end of testing, all the LIVs for that device are assumed to come from an operational device. For the set of QCLs part of the 150-hour burn-in process, there are 444 individual LIV measurements in total. 95 of the LIVs are from devices that fail during testing. The remaining 349 LIV measurements are from devices that are operational at the end of testing.

The RBF-SVM has two hyperparameters that are adjusted during training to achieve high classification accuracy. To tune these hyperparameters, we employ a grid-search algorithm, where we vary the parameters between $10^{-3}$ to $10^{3}$ with an adaptive step size, yielding a parameter matrix of 72 rows by 72 columns. For each pair of hyperparameters, we calculate the accuracy of the SVM. To avoid overfitting to a particular set of training data, we repeat this process for 10 different sets of training data. Each of the 10 training data sets consists of LIVs from four operational devices and one failed device. A score is assigned to each pair of hyperparameters, by averaging the classification accuracy scores of the 10 cases. In the end, the SVM with the hyperparameter pair yielding the highest score is selected for use in the model. We emphasize that even though we use different combinations of devices in the training and test sets, we never mix the two sets in any experiment. In other words, the SVM is never trained on the devices in the test set. When using the RBF-SVM to classify the LIVs for the two devices that underwent the longer burn in process, we train the SVM using $\left(G_{i} ; i=1 . .5, B_{j} ; j=1,2\right)$. The RBF-SVM uses the same values for the hyperparameters that were determined previously.

\section{Results And Discussion}


Figure 3 shows the predictions of the model for different combinations of test data. Here, for each combination, the algorithm was trained on the LIVs from the remaining five devices (four operational devices and one failed device) and tested using the indicated operational (G) and failed (B) devices. For example, for Combination 1, LIVs from G2, G3, G4, G5 and B2 are used for training the SVM and LIVs from devices $\mathrm{G} 1$ and $\mathrm{B} 1$ are used for testing the SVM. Each colored rectangle represents a prediction made by the SVM for the LIV measured at the indicated time. The time of the QCL failure is indicated with a black cross.

As shown in Fig. 3, the model identifies healthy devices with $100 \%$ accuracy for every test combination, encompassing over 1500 hours of measurements for the operational devices. Furthermore, for each device that fails during the burn-in testing, the SVM categorizes multiple LIVs as belonging to a failed device many measurements (hours) before the actual failure of the QCL. In some instances, the first measured LIV is categorized as belonging to a failed device. With no false negatives (identifying an LIV from an operational device as belonging to a failed device) observed, high confidence can be assigned to predicting devices will fail even from a single LIV that is categorized as coming from a failed device.

We use the output of the SVM to classify the QCL as healthy or failing. A device is classified as failing at a measurement interval if any of the LIV measurements up to that point in time are identified as belonging to a device that will fail (red boxes in Fig. 3). Otherwise, the device is classified as healthy. To evaluate the SVM based classifier, we calculate the specificity and sensitivity at each of the measurement intervals. The specificity, also known as true positive rate, is the ratio of the number of devices predicted as healthy over the total number of healthy devices, i.e., operational devices at the conclusion of the burnin process. The sensitivity is the ratio of the number of devices predicted as failing over the total number of devices that fail during the burn-in process. For the seven QCLs scheduled for the shorter burn-in time, the average specificity is $100 \%$ and the average sensitivity for the 10 cases is $93.65 \%$ across all the hours. In Fig. 4, the change of sensitivity and specificity with respect to the burn-in time is depicted. A sensitivity greater than $80 \%$ is obtained after 8 hours of burn-in time. Furthermore, for every combination of test and training data, the specificity is constant at $100 \%$. For these QCLs, premature failure can be predicted as much as 129 hours before the actual failure of the device.

Two of the devices were scheduled for longer burn-in periods of 250 hours. However, both devices failed before the completion of the burn-in testing. Figure 4 shows the results of classifying the measured LIVs from these devices. For device B3, the first LIV classified as belonging to a device that will fail occurs 13 hours before the failure of the QCL. For device B4 however, the second measured LIV indicates that the device will fail prematurely, 205 hours before the actual device failure. While the lead times between identifying the QCL as a device that will fail and the actual failure for these two devices are different, the SVM classifies at least one LIV as belonging to a failing device for each of the lasers. We speculate that the differences for these two devices could be related to the testing conditions. One possibility is that the device B3 was damaged during testing, for example from a brief power surge, and that damage ultimately resulted in the observed failure, whereas device B4 had underlying problems from the beginning of testing and would eventually exhibit premature failure. 
Classifying LIVs using a SVM could have significant impact beyond predicting the premature failure of individual devices, and this is highlighted by the differences in the classification of the LIVs for devices B3 and B4. As more devices undergo the accelerated burn-in process and LIV measurements are classified, it will be possible to develop an understanding of the timescale for the failure process. In fact, the effectiveness of this approach using LIV measurements separated by three-hour intervals already gives important information about the timescale for the failure process. We expect that incorporating additional devices and measurements into this work will lead to further improvements in QCLs and new capabilities for filtering short-lived devices from commercial inventory.

\section{Conclusions And Future Work}

This paper presents an accelerated burn-in process that incorporates a classifier based on a supervised learning algorithm for predicting premature device failure. The hyperparameters for the SVM-RBF are determined using multiple training data sets to minimize the possibility of overfitting. The presented findings indicate that operational QCLs are always identified correctly without any false negatives. In addition, all QCLs that fail have at least one LIV that indicates that it will fail. Collectively, this gives us high confidence that a LIV labeled as coming from a failing device indicates that there will be premature failure of that device. The SVM classifies some of the QCLs as devices that will exhibit premature failure based on the first LIV measurement, which can drastically reduce time spent on burn-in processes for devices that will fail. In the best case, this is 205 hours before the actual failure.

Our approach to using a SVM-based classifier for predicting premature QCL failure can be extended to QCLs with different active region and waveguide designs. Additionally, the ability of the existing classifier to predict premature failure up to 200 hours before the actual device failure could provide direction for future research into the origins of premature failure in QCLs. Finally, the strategy of employing a SVMbased classifier for predicting premature failure can be incorporated into the QCL manufacturing process to decrease the overall cost of ownership.

\section{Declarations}

Acknowledgements

This work was supported in part by the Department of the Navy, Award Number: N68936-21-C-0007 Author contributions

A.J.H. and C.A. conceived the concept of the paper. S.J. conducted the burn-in measurements and C.A. analyzed the data and developed the SVM classifier. S.J., X.W., M.F., M.T., and A.J.H. directed the burn-in measurements. V.G. and A.J.H. oversaw the machine learning work. C.A. and A.J.H. wrote the manuscript and all authors reviewed the manuscript.

Data Availability 
The data that support the plots within this paper and other findings of this study are available from the corresponding authors upon reasonable request.

Competing interests

The author(s) declare no competing interests.

Corresponding author

Correspondence to Cagri Aydinkarahaliloglu.

\section{References}

1. Yao, Y., Hoffman, A. J. \& Gmachl, C. F. Mid-infrared quantum cascade lasers. Nat. Photonics 6, 432439 (2012).

2. Howard, S. S. et al. High-Performance Quantum Cascade Lasers: Optimized Design Through Waveguide and Thermal Modeling. IEEE J. Sel. Top Quant 13, 1054-1064 (2007).

3. Liu, P. Q. et al. Highly power-efficient quantum cascade lasers. Nat. Photonics 4, 95-98 (2010).

4. Villares, G., Hugi, A., Blaser, S. \& Faist, J. Dual-comb spectroscopy based on quantum-cascade-laser frequency combs. Nat. Commun. 5, 5192 (2014).

5. Gmachl, C., Capasso, F., Sivco, D. L. \& Cho, A. Y. Recent progress in quantum cascade lasers and applications. Rep. Prog. Phys. 64, 1533 (2001).

6. Hugi, A., Villares, G., Blaser, S., Liu, H. C. \& Faist, J. Mid-infrared frequency comb based on a quantum cascade laser. Nature 492, 229-233 (2012).

7. Lyakh, A., Suttinger, M., Go, R., Figueiredo, P. \& Todi, A. $5.6 \mu \mathrm{m}$ quantum cascade lasers based on a two-material active region composition with a room temperature wall-plug efficiency exceeding $28 \%$. Appl. Phys. Lett. 109, 121109 (2016).

8. Babichev, A. V. et al. High-Power Quantum-Cascade Lasers Emitting in the 8- $\mu \mathrm{m}$ Wavelength Range. Tech. Phys. Lett+ 45, 735-738 (2019).

9. Capasso, F. High-performance midinfrared quantum cascade lasers. Opt. Eng. 49, 111102-111102-9 (2010).

10. Hundt, P. M. et al. Multi-species trace gas sensing with dual-wavelength QCLs. Appl. Phys. B 124, 108 (2018).

11. Mezzapesa, F. P. et al. Continuous-Wave Reflection Imaging Using Optical Feedback Interferometry in Terahertz and Mid-Infrared Quantum Cascade Lasers. IEEE T. Thz Sci. Techn. 4, 631-633 (2014).

12. Day, T. et al. Quantum cascade lasers for defense and security. Proc. Spie 889802-889802-8 (2013) doi:10.1117/12.2031536.

13. Evans, A. \& Razeghi, M. Reliability of strain-balanced Ga0.331 In0.669As/Al0.659In0.341As/InP quantum-cascade lasers under continuous-wave room-temperature operation. Appl. Phys. Lett. 88, 261106 (2006). 
14. Myers, T. L., Cannon, B. D., Taubman, M. S. \& Bernacki, B. E. Performance and reliability of quantum cascade lasers. Proc. Spie. 87330E-87330E-15 (2013) doi:10.1117/12.2015479.

15. Xie, F. et al. Long-term reliability study and failure analysis of quantum cascade lasers. Nov. In-plane Semicond. Lasers Xvi 101231J-101231J-10 (2017) doi:10.1117/12.2255069.

16. Myers, T. L. et al. Long-term operational testing of quantum cascade lasers. Micro- Nanotechnol. Sensors Syst. Appl. Viii 98362J-98362J-11 (2016) doi:10.1117/12.2223129.

17. Knipfer, B. et al. Failure Analysis of High-Power (One-Watt) Room-Temperature Continuous Wave MOCVD Quantum Cascade Lasers. 2018 IEEE Int. Semicond. Laser Conf. Is/c. 1-2 (2018) doi:10.1109/islc.2018.8516151.

18. Xie, F. et al. Reliability of 4.6- $\mu \mathrm{m}$ quantum cascade lasers under continuous-wave room-temperature operation. Opt. Eng. 49, 111104-111104-5 (2010).

19. Jordan, M. I. \& Mitchell, T. M. Machine learning: Trends, perspectives, and prospects. Science 349, 255-260 (2015).

20. Otter, D. W., Medina, J. R. \& Kalita, J. K. A Survey of the Usages of Deep Learning for Natural Language Processing. IEEE T. Neur. Net. Lear. 32, 604-624 (2021).

21. Noda, K., Yamaguchi, Y., Nakadai, K., Okuno, H. G. \& Ogata, T. Audio-visual speech recognition using deep learning. Appl. Intell. 42, 722-737 (2015).

22. Voulodimos, A., Doulamis, N., Doulamis, A. \& Protopapadakis, E. Deep Learning for Computer Vision: A Brief Review. Comput. Intel. Neurosc. 2018, 7068349 (2018).

23. Noble, W. S. What is a support vector machine? Nat. Biotechnol. 24, 1565-1567 (2006).

24. Cortes, C. \& Vapnik, V. Support-vector networks. Mach. Learn. 20, 273-297 (1995).

25. Wang, Y., Zhang, M., Tang, X., Peng, F. \& Yan, R. A kMap optimized VMD-SVM model for milling chatter detection with an industrial robot. J. Intell. Manuf. 1-20 (2021) doi:10.1007/s10845-02101736-9.

26. Liu, C., Li, Y., Zhou, G. \& Shen, W. A sensor fusion and support vector machine based approach for recognition of complex machining conditions. J. Intell. Manuf. 29, 1739-1752 (2018).

27. Guyon, I., Weston, J., Barnhill, S. \& Vapnik, V. Gene Selection for Cancer Classification using Support Vector Machines. Mach. Learn. 46, 389-422 (2002).

28. Souza, J. da S., Santos, M. V. L. dos, Bayma, R. S. \& Mesquita, A. L. A. Analysis of Window Size and Statistical Features for SVM-based Fault Diagnosis in Bearings. IEEE Lat. Am. T. 19, 243-249 (2021).

29. Xu, Y., Xu, Y. \& Li, W. Fault Diagnosis for Gearbox Based on Genetic-SVM Classifier. 2010 2nd Int. Conf. Comput. Automation Eng. Iccae. 1, 361-363 (2010).

30. Pedregosa, F. et al. Scikit-learn: Machine Learning in Python. Arxiv (2012).

\section{Figures}




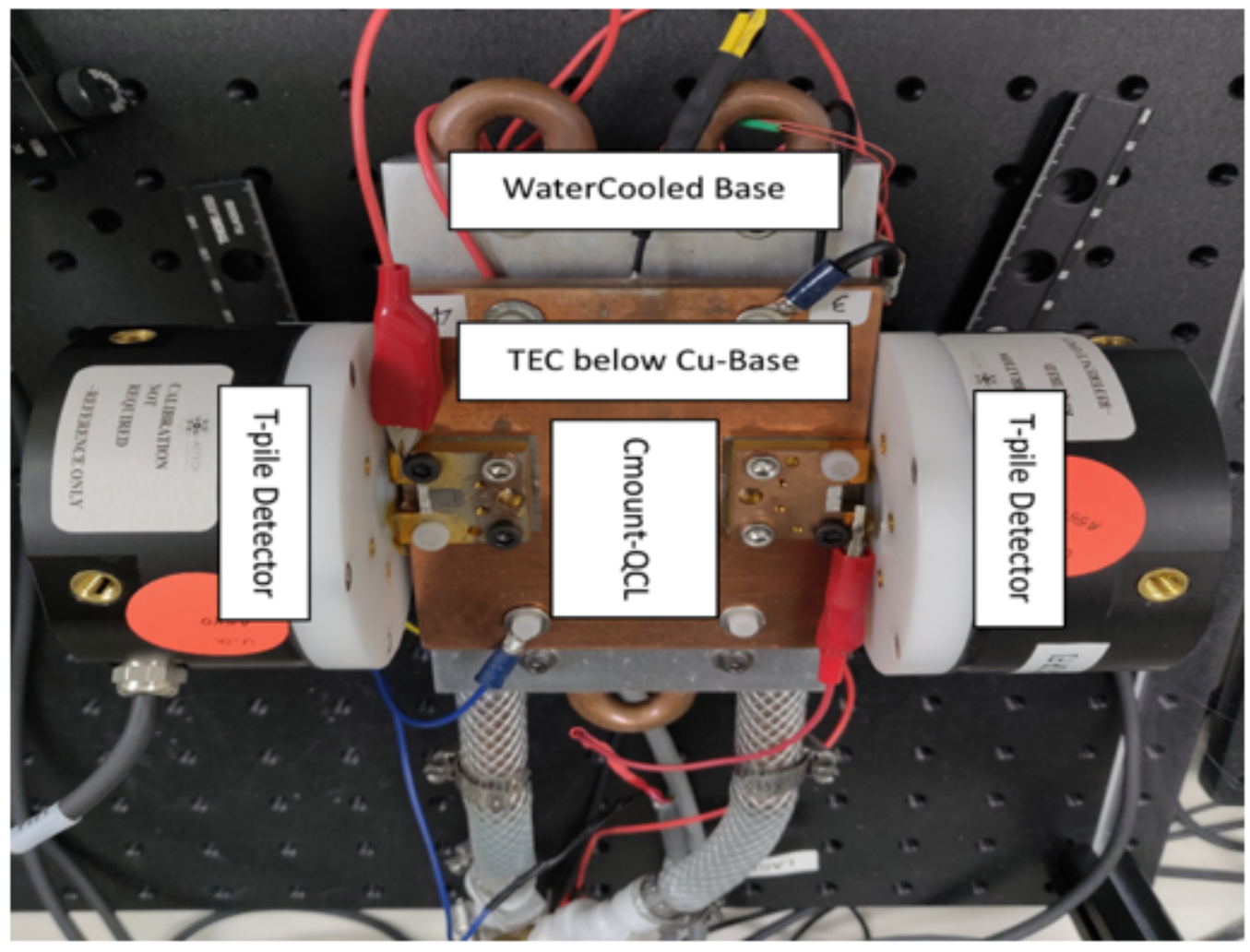

Figure 1

QCL Mount with integrated water-cooled TEC for high temperature accelerated lifetime testing.
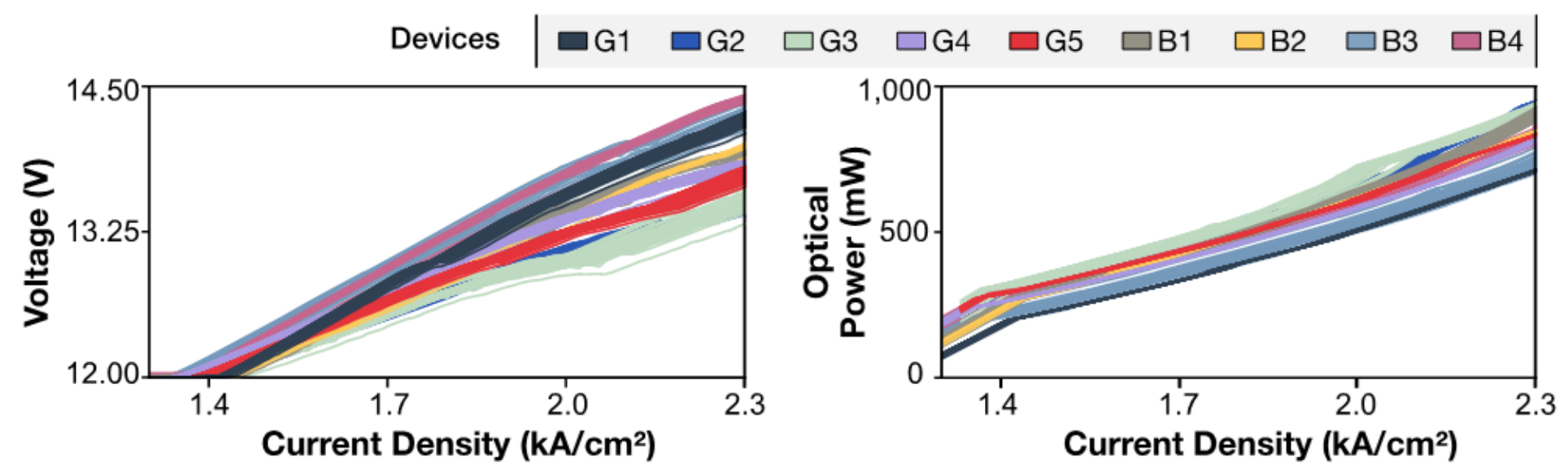

Figure 2

Current density versus voltage (left) and current density versus optical power (right) characteristics for the nine devices. After burn-in, the devices that were still functional are denoted with G1, G2, G3, G4 and G5, whereas defective devices are denoted as B1, B2, B3 and B4. Device condition identification by eye is not apparent. 


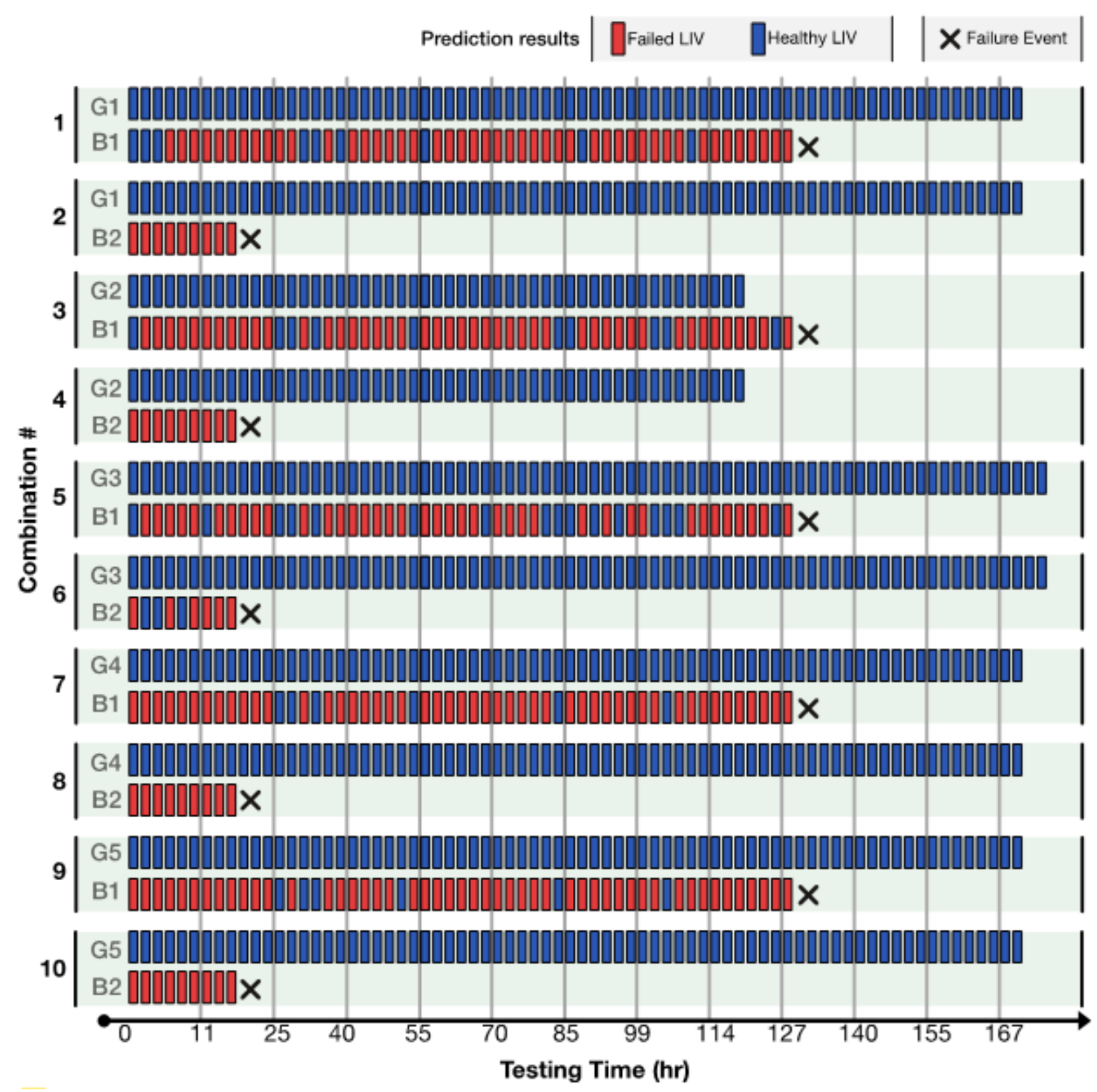

Figure 3

SVM testing results for the first stage of experiments. Rectangles represent measurement events at different time points, while their color represent the model's prediction. The failure event is marked with a black cross. The SVM model predicts failure for all of the defective devices up to 127 hours prior to the failure event. 

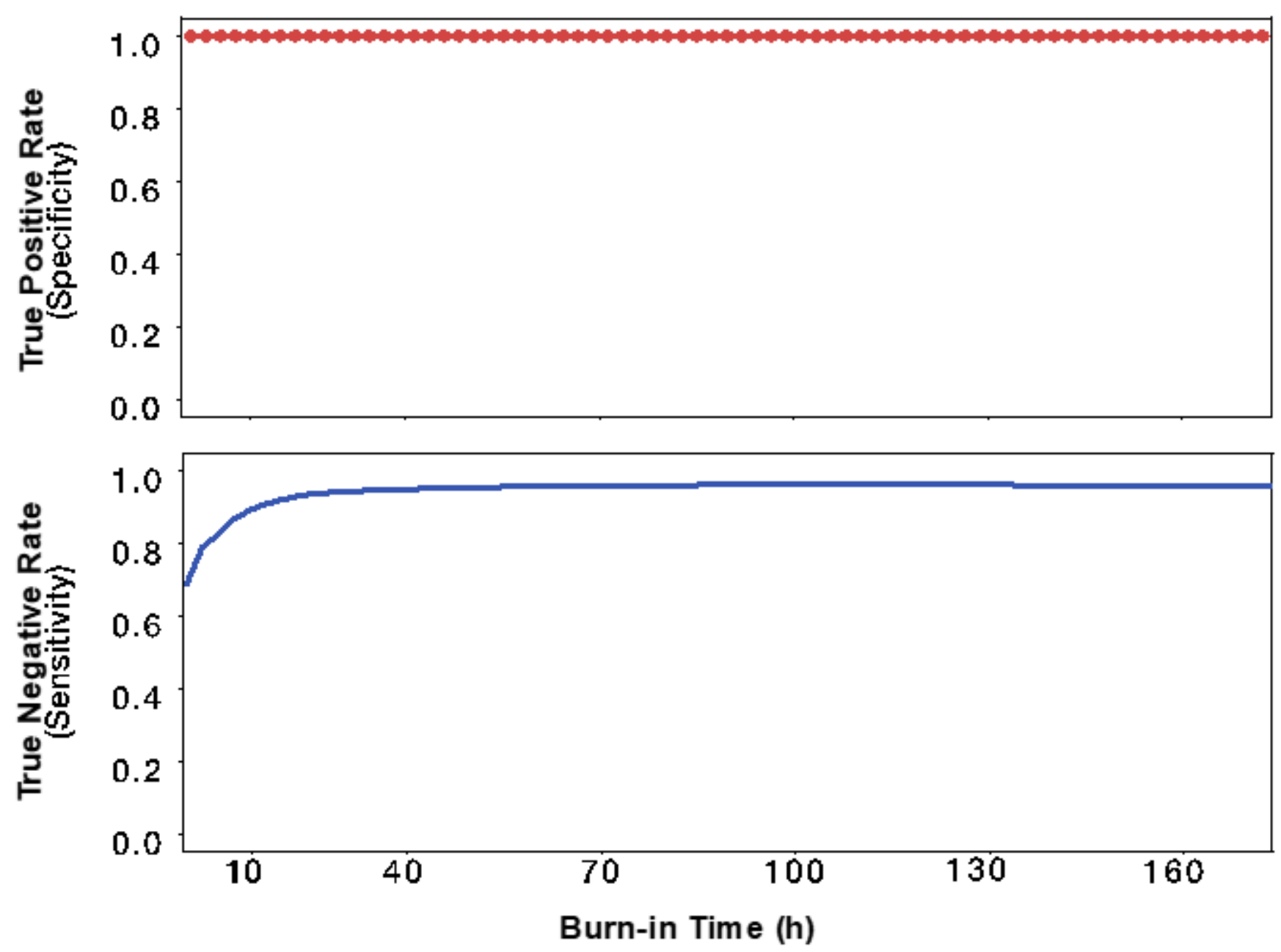

Figure 4

Specificity and sensitivity with respect to hours before failure. Blue shaded region indicates the error margin for all the cases. 


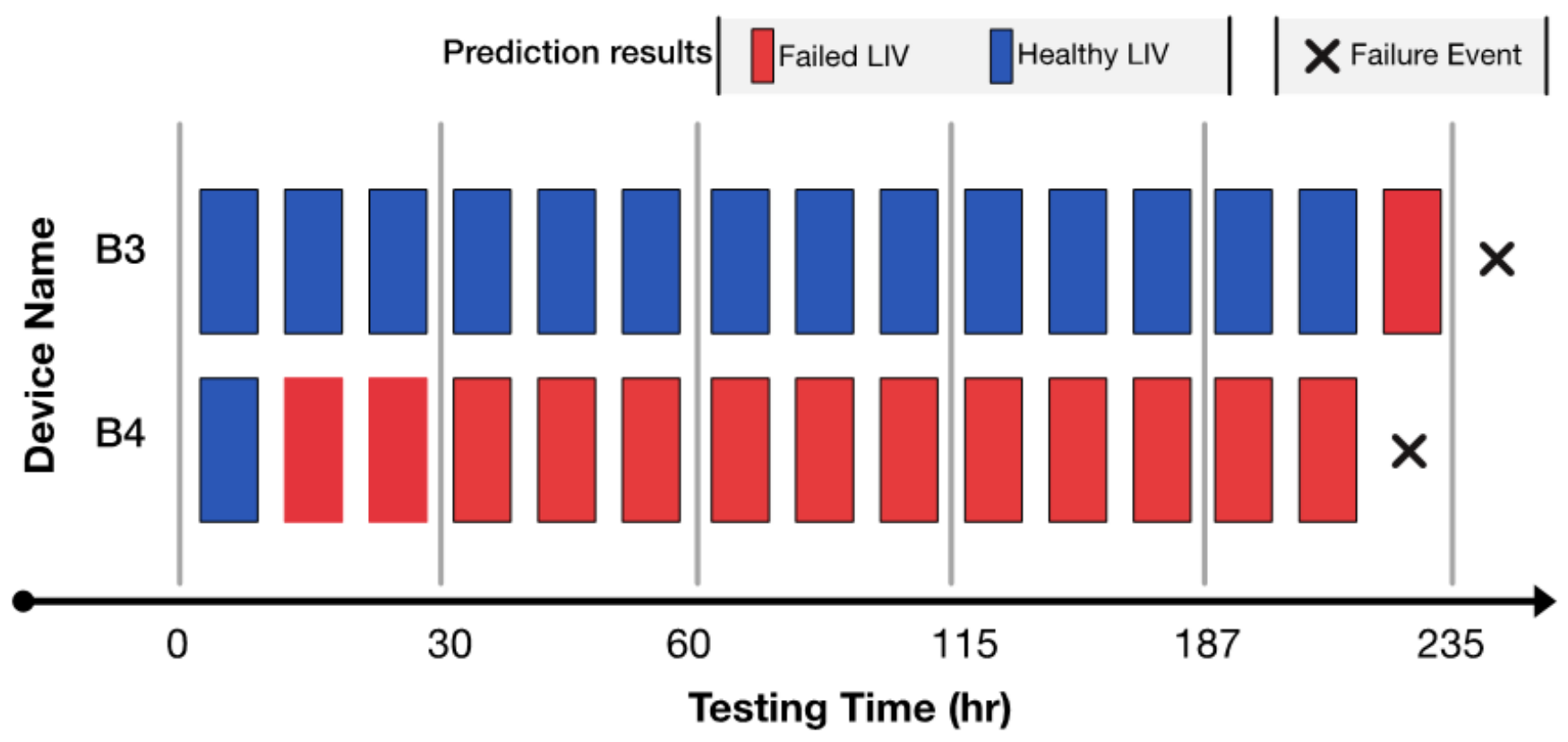

Figure 5

SVM testing results for the second stage of experiments. Rectangles represent measurement events at different time points, while their color represent the model's prediction. The failure event is marked with a black cross. 